SLAC-PUB-8100

June 1999

updated September 2000

\title{
High-Power Vacuum Window in WR10
}

\author{
Marc E. Hill, Richard S. Callin and David H. Whittum \\ Stanford Linear Accelerator Center, Stanford University, Stanford, CA 94309
}

\begin{abstract}
Results are presented for fabrication and test of a WR10 waveguide window, for use in ultra-high vacuum at $91.4 \mathrm{GHz}$. Low-power bench measurements are compared with analytic and simulation results. Operation at $\approx$ to $4 \mathrm{~kW}$ peak power, duty factor $10^{-6}$ and $10^{-9}$-scale vacuum is noted.
\end{abstract}

Submitted to IEEE Transcations on Microwave Theory and Techniques

Work supported by the Department of Energy contract DE-AC03-76SF00515 


\title{
High-Power Vacuum Window in WR10
}

\author{
Marc E. Hill, Richard S. Callin and David H. Whittum
}

\begin{abstract}
Results are presented for fabrication and test of a WR10 waveguide window, for use in ultra-high vacuum at 91.4 GHz. Low-power bench measurements are compared with analytic and simulation results. Operation at $\approx 4 \mathrm{~kW}$ peak power, duty factor $10^{-6}$ and $10^{-9}$-scale vacuum is noted.
\end{abstract}

In a microwave accelerator network, waveguide windows are employed to isolate the $10^{-7}$-torr beamline vacuum from the $10^{-9}$-torr vacuum of the power tube. The window is essential to long tube-life, currently in the range of $5 \times 10^{4}$ hours, for the 65 -MW klystrons powering the TwoMile Accelerator (TMA) [1]. Future accelerators will require shorter wavelengths to reach higher gradients, and research toward a $\mathrm{GV} / \mathrm{m}$ linac presently concentrates on W-Band, at the 32nd harmonic of the $2856 \mathrm{MHz}$ TMA operating frequency [2]. Engineering of such a miniature mmwave linac requires development of a new class of $\mathrm{mm}$-wave components, compatible with high-vacuum, and capable of handling high-peak power, albeit at low duty cycle. Here we report results of bench and high-power tests of such a window, the first test of such a mm-wave assembly in a working accelerator.

Conceptually the window consists of WR10 waveguide, operated in fundamental $T E_{10}$-mode, and a slab of dielectric filling a length of the guide. Wave transmission through a length $L$ of lossless dielectric is described by

$$
\left|S_{21}\right|^{2}=\left[\cos ^{2} \beta^{\prime} L+\frac{1}{4}\left(\frac{\beta}{\beta^{\prime}}+\frac{\beta^{\prime}}{\beta}\right)^{2} \sin ^{2} \beta^{\prime} L\right]^{-1}
$$

where $\beta$ and $\beta^{\prime}$ are the guide wavenumbers in the vacuum and dielectric portions of the guide,

$$
\beta=\frac{2 \pi}{\lambda} \sqrt{\mu_{r} \varepsilon_{r}-\left(\frac{\lambda}{2 a}\right)^{2}},
$$

$\lambda$ is the free-space wavelength, $a$ is the waveguide width $(0.10 ")$, and $\mu_{r}$ and $\varepsilon_{r}$ are the relative permeability and permittivity of the dielectric. For a good match, the window length should be a multiple of half the dielectric guide wavelength, or $\beta^{\prime} L=n \pi$, with $n$ an integer. In addition, Eq. (1) implies that highest bandwidth is attained with the shortest window length. We selected an $n=2$ or " $1-\lambda$ " window design as a compromise between bandwidth and ease of assembly.

As to assembly, the $0.100 " \times 0.050$ " oxygen-free highconductivity (OFE) copper WR10 rectangular waveguide

Work supported by the U.S. Department of Energy under contract DE-AC03-76SF00515.

M.E. Hill is with the Physics Department at Harvard University, Cambridge, MA 02138.

R.S. Callin and D.H. Whittum are with the Stanford Linear Accelerator Center, Stanford University, Stanford, California 94309.

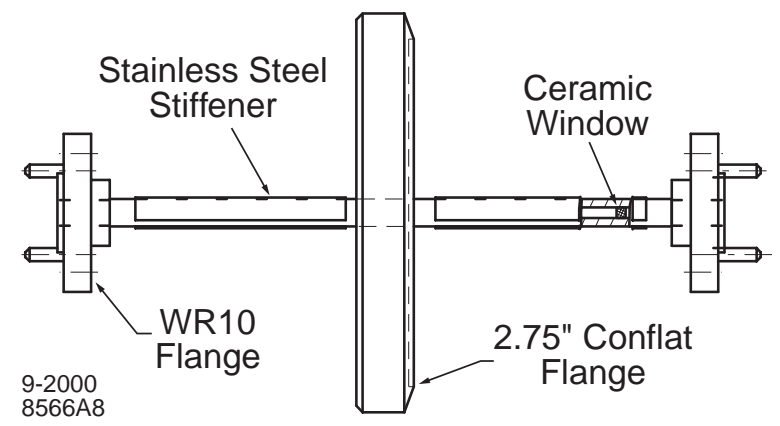

Fig. 1. Mechanical drawing of the final window assembly including rf and vacuum flanges.

is obtained unflanged from commercial extruded stock and chemically cleaned. The dielectric consists of WesCo AL995 alumina ceramic, sliced and ground from a 3" disk, into rods of dimension $.098^{\prime \prime} \times .048$ " $\times 2$ ". The rods are coated with a 1-mil layer of molybdenum-manganese and fired at $1500^{\circ} \mathrm{C}$ forming a metallized layer for brazing. The windows are then cut to their final length of .0428 " and brazed into the OFE waveguide. The waveguide flanges are attached in a separate step. They consist of 304L VAR (vacuum-arc remelt) stainless steel, machined to the standard 3/4" circular WR10 waveguide flange geometry. These are attached with a second, lower-temperature braze. After each braze step, the window is leak-checked to ensure vacuum integrity.

One concern with this process is the difference in the thermal expansion coefficient of the ceramic $\left(9.3 \times 10^{-6}\right)$ and the copper waveguide $\left(17 \times 10^{-6}\right)$. Differential expansion during the $1000^{\circ}$ braze cycle may in principle produce a gap as large as 1 mil between the dielectric and copper surfaces. For lower frequency, larger-dimension window assemblies, such differential expansion may be restrained by a molybdenum retaining wire encircling the ceramic window assembly. We omitted this step for the W-Band assembly, and encountered no problems, although one might expect that its use would produce a smaller braze fillet.

The completed window-assembly was subjected to transmission and reflection measurements in WR10 using a custom-built vector network analyser described elsewhere [3]. The results, seen in Fig. 2, were fit with the analytic result of Eq. (1), employing $\varepsilon_{r}$ as a fit parameter. We infer $\varepsilon_{r} \approx 9.485 \pm .03$ at $91.4 \mathrm{GHz}$. Also seen in Fig. 2 is the result of numerical simulation via the finite-difference code, GdfidL [4], giving good agreement.

One additional concern in this geometry is the presence of higher modes, "ghost modes", in the overmoded dielectric guide. One is particularly concerned to avoid a spurious resonance in the operating band and the added insertion loss that would result. Fig. 2 shows the power sum 


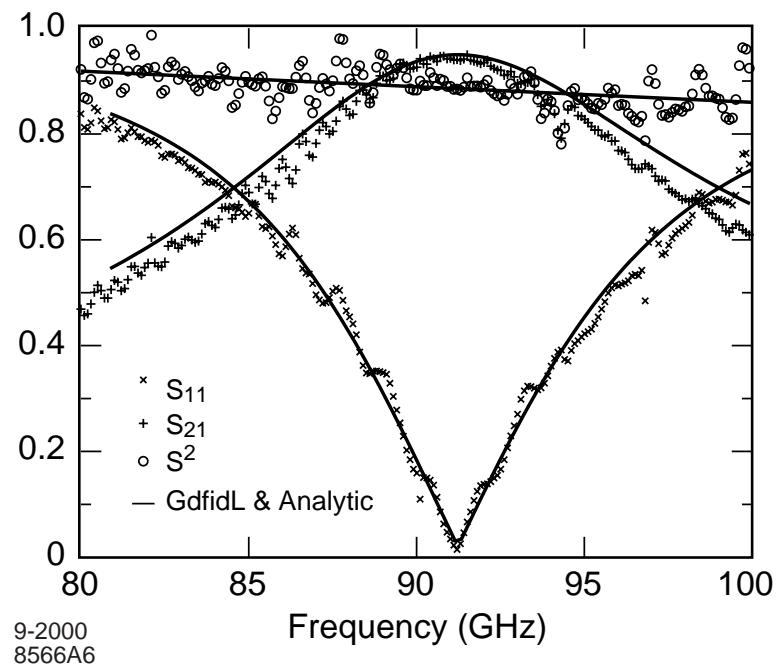

Fig. 2. Plot of measured scattering parameters, $S_{11}$ and calculated scattering parameters for a sample window. $S^{2}=S_{11}^{2}+S_{21}^{2}$.

$\left|S_{11}\right|^{2}+\left|S_{21}\right|^{2}$, from which we can determine the insertion loss, and inspect the operating band for ghost-modes. The observed attenuation of $0.2 \mathrm{~dB}$ seen in Fig. 2 is consistent with loss through the 3 " copper waveguide. With such a short length of ceramic, results are not sensitive to a loss tangent in the $10^{-4}$ range.

After bench measurements, this window was employed on the output of a test cavity installed on a microwave accelerator at SLAC. This experiment, to be described in a later publication, produced peak power levels of $4 \mathrm{~kW}$. This corresponds to a maximum electric field in the waveguide of $1.5 \mathrm{MV} / \mathrm{m}$. The window was an essential component for this test as the solid-state components needed for power detection are not vacuum compatible, and, in any case, are most conveniently situated outside the vacuum envelope.

The design and the process appear to be mechanically sound, and robust; following the first working window, several additional windows have been fabricated, and their rf characteristics are in close agreement with the foregoing, while they have all passed leak check. Thus far we have seen no evidence of breakdown, multipactor, fracture, or puncture even while realizing that such phenomena are part of the experiment, and part of the utility of the apparatus in the first place.

To conclude, we have begun to develop vacuumcompatible components in WR10, for operation with a miniature accelerator. The immediate and initial purpose for the window was to permit monitoring of power developed in a sub-harmonic interaction circuit. Alternatives to a window include coaxial feedthrough, horn output through a window, taper to oversize guide and window. However, all of these techniques have their own challenges, and even if successful, leave one with a more complicated power calibration. More than that, they are not ideally suited for accelerator component development. With a WR10 window we can couple power to other WR10 components in a straightforward way. We expect that development of more sophisticated, quasi-optical accelerator components will later benefit from the calibrations and other work facilitated by this simple, yet robust window design.

This work is indebted to the support and encouragement of G. Caryotakis and J. Huth, helpful conversations with W.R. Fowkes, and the expert assistance of D. Miller and D. Millican.

\section{REFERENCES}

[1] G. Caryotakis, "High Power Microwave Tubes: In the Laboratory and On-Line", IEEE Trans. Plasma Sci. 22, pp. 683-691, 1994.

[2] D.H. Whittum, "Ultimate gradient in solid-state accelerators", Proceedings of the Advanced Accelerator Concepts Workshop (AIP, to be published), SLAC-PUB-7910.

[3] R.H.Siemann, "W-Band vector network analyzer based on an audio lock-in amplifier", Phys. Rev. ST-AB (to be published), SLAC-PUB-7884.

[4] W. Bruns, "GdfidL: A finite difference program for arbitrarily small perturbations in rectangular geometries", IEEE Trans. Magn. 32, pp. 1453-1456, 1996. 